\title{
ENHANCED PRODUCT QUALITY \& ENERGY EFFICIENCY BY MEANS OF INTEGRATED PLANT MONITORING SYSTEM*
}

\author{
Detlef Ehlert ${ }^{1}$ \\ Alfred Metzul \\ Martin Bläsner ${ }^{3}$
}

\begin{abstract}
In view of the increasing level of cost pressure on the part of plant owners, optimized plant operation with top product quality and maintenance costs is indispensable. Unscheduled shutdowns with increased repair costs and production downtimes are significantly reduced by preventive maintenance and the stock-keeping of spare parts. To remain competitive in the steel market, it is important for plants owners to operate their plants with the highest possible degree of energy efficiency and at high availability in order to manufacture high-quality products. Plant Monitoring Systems make it possible to optimize the manner of production. An integrated overview of the overall process, under consideration of process stability, product quality, production costs and plant availability ensures a "transparent plant".
\end{abstract}

Keywords: Plant asset management; Condition monitoring; Preventive maintenance; Quality monitoring.

1 Dipl.-Ing, Executive Vice President, Electrical and Automations Systems, SMS Group, Hilchenbach, NRW, Germany

2 Dipl.-Ing., General Manager, Electrical and Automation Systems/Automation Level 1 Flat Rolling Mills, SMS Group, Hilchenbach, NRW, Germany

3 Dr. rer. nat., Process Engineer, Electrical and Automation Systems/Process Models Metallurgical Plants, SMS Group, Düsseldorf, NRW, Germany 


\section{INTRODUCTION}

Strongly contested markets and globalized competition are resulting in an ever higher level of cost pressure on the part of steelworks owners. To remain competitive in this market, it is important that our customers should operate their plants with the highest possible degree of energy efficiency and with high availability in order to manufacture very high-quality products. It is therefore indispensable to ensure optimization of the plant availability and enhanced process efficiency while considering the customers' requirements as regards the products.

To support plant owners in this, SMS Siemag has developed an integrated Monitoring System comprising modules for the monitoring of

- Product quality;

- Energy efficiency;

- Plant condition.

These three modules allow an integrated overview of the overall process, under consideration of process stability, product quality, production costs and plant availability.

Figure 1 shows the implementation of the Integrated Monitoring System under consideration of these requirements regarding optimized plant operation.

The modular structure offers two major advantages: The system can be utilized both for individual plant sections and for integrated plants. Furthermore, only individual modules can be utilized, e.g. for the monitoring of energy consumption. By means of configurable allocation of roles, the system enables results to be examined from various viewpoints, e.g. from those of the maintenance engineer or the production planner.

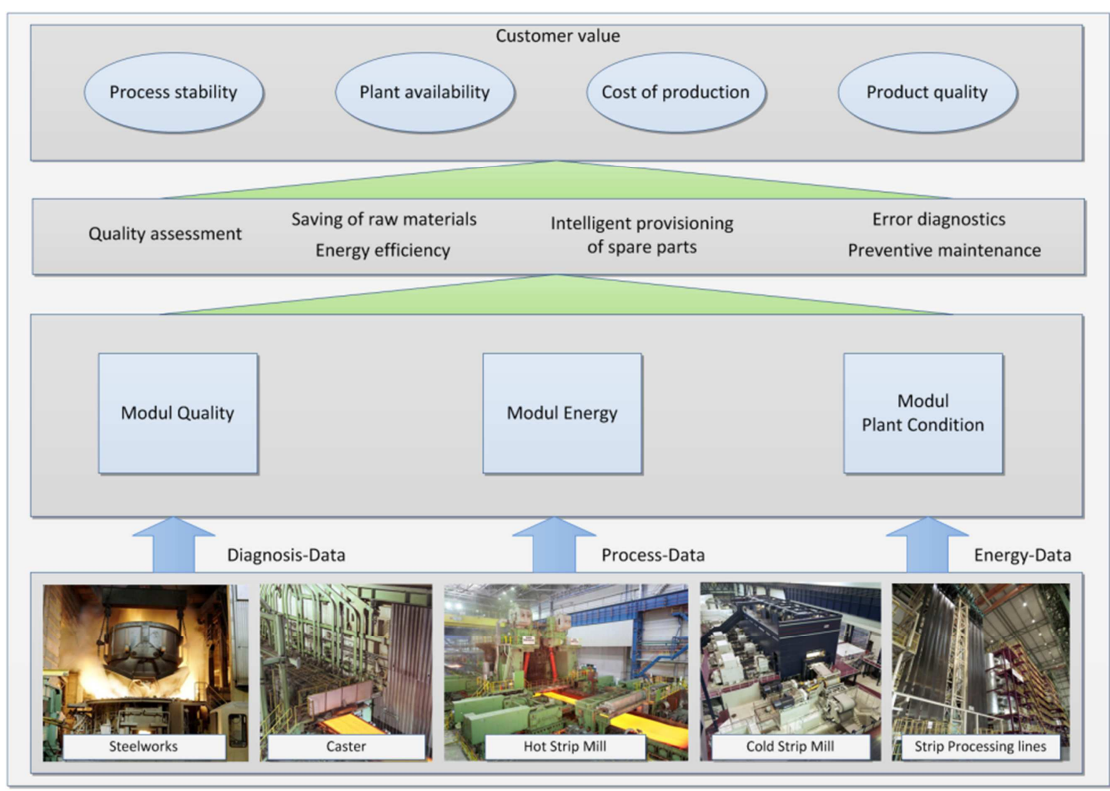

Figure 1: Overview of the Integrated Monitoring System

In the following, the individual modules for monitoring of product quality, energy efficiency and plant condition are introduced. 


\section{MODULS OF THE INTEGRATED MONITORING SYSTEM}

\subsection{Product Quality Module}

The Quality Monitoring module known as X-Pact ${ }^{\odot}$ Product Quality Analyzer allows, in a manner covering all process stages, the examination and processing of plant and process data and their relationships with the product characteristics (see

Figure 2). In this way, continuous quality assurance and process optimization is made possible.

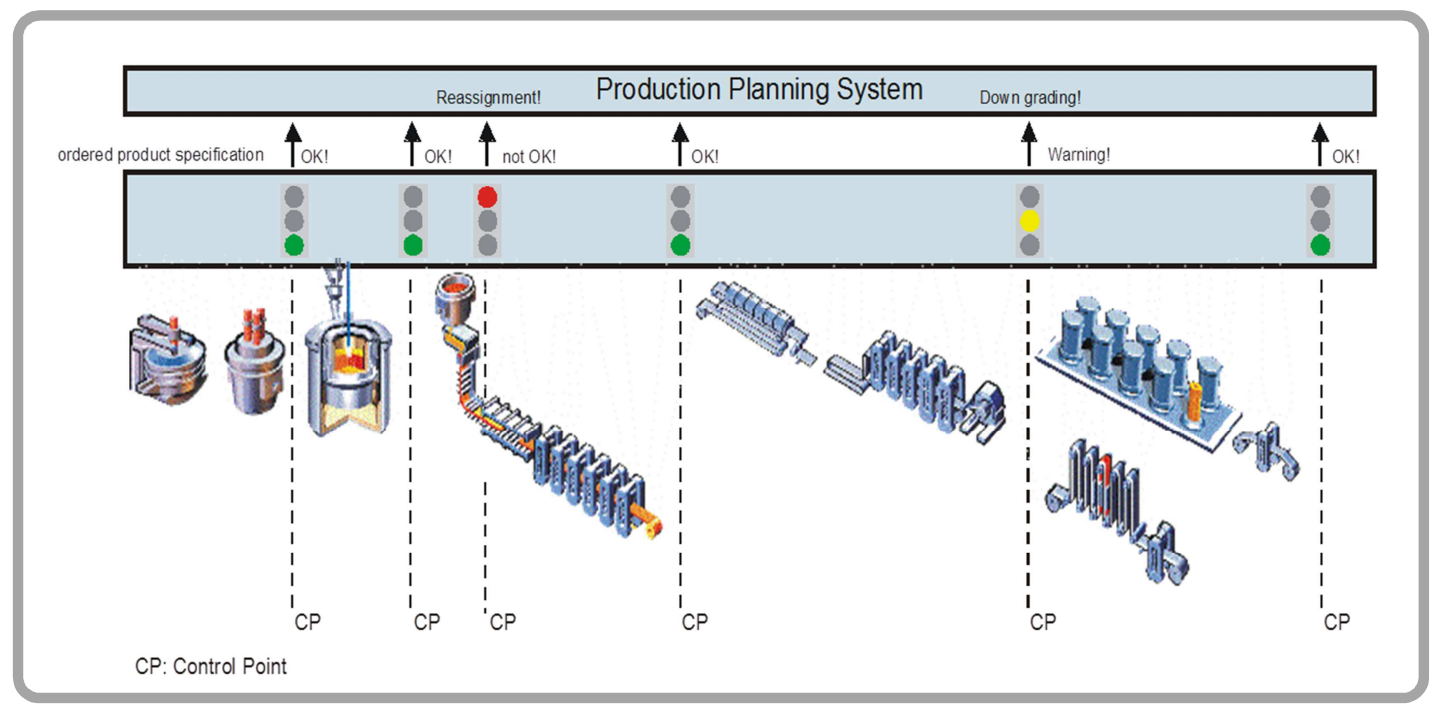

Figure 2: Monitoring of the various process stages

The module basically comprises a quality database which contains all process, measurement and surface-inspection data relevant to quality as well as data generated with the aid of quality models. These data remain available over a long period in compressed and product-oriented form. This enables comparisons to be made and any deterioration of the product quality to be detected.

Figure 3 shows as an example a fault analysis in which an interrelationship between thickness measurements of the strip in different production steps is examined. The length-related data of the thickness measuring systems are evaluated here with the aid of the data allocated to them. 


\section{9th Industrial $\Pi$ and Automation}

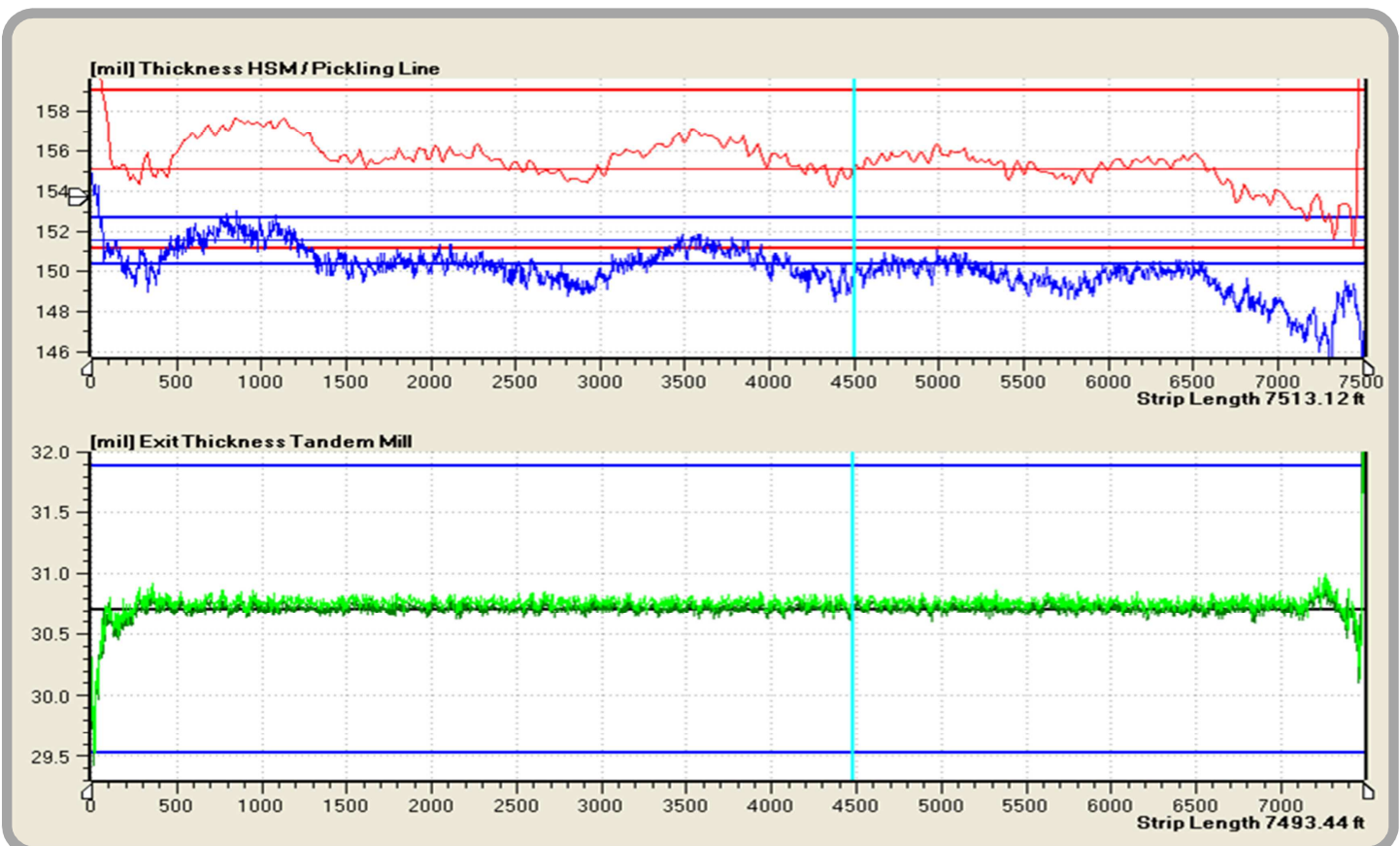

Figure 3: Evaluation of quality-relevant data such as those relating to the thickness measurements in the hot rolling mill, pickling line and tandem cold mill

The integrated quality monitoring system offers extensive benefits to the operation of a plant:

- Optimized production

- Reduction of production cost

- Higher output

- Optimized complaint management

- Less complaints

A further module belonging to the Integrated Monitoring System is the energy efficiency module known as the X-Pact ${ }^{\circledR}$ Energy Advisor.

\subsection{Energy Efficiency Module}

Optimized plant operation is necessary nowadays in view of rising energy costs, limited availability of raw materials and stipulations relating to environmental policy. The $\mathrm{X}-\mathrm{Pact}^{\odot}$ Energy Advisor assists the plant owner in lowering energy costs and keeping them stable. The continuous recording of all energy-relevant plant data enables potential savings to be identified and efficiently implemented. The interlinking of energy data with process, product and plant condition data allows a clear representation of influencing variables and derived characteristics. Thus, for example, with the aid of a "speedometer", the current process status and the energy efficiency can be rapidly recorded and evaluated (see Figure 4).

The X-Pact ${ }^{\odot}$ Energy Advisor offers a large number of further possibilities, such as:

- Generation of improvement projects

- Comparison with results from previous periods

- Indication of trends regarding energy consumption

- Planning of energy requirement notifications / Coordination with energy supply company

- 15-minute anticipatory calculation of mean value in order to observe contractual consumption limits 
- Display and recording of energy and production variables

- Early detection of production defects and machine wear

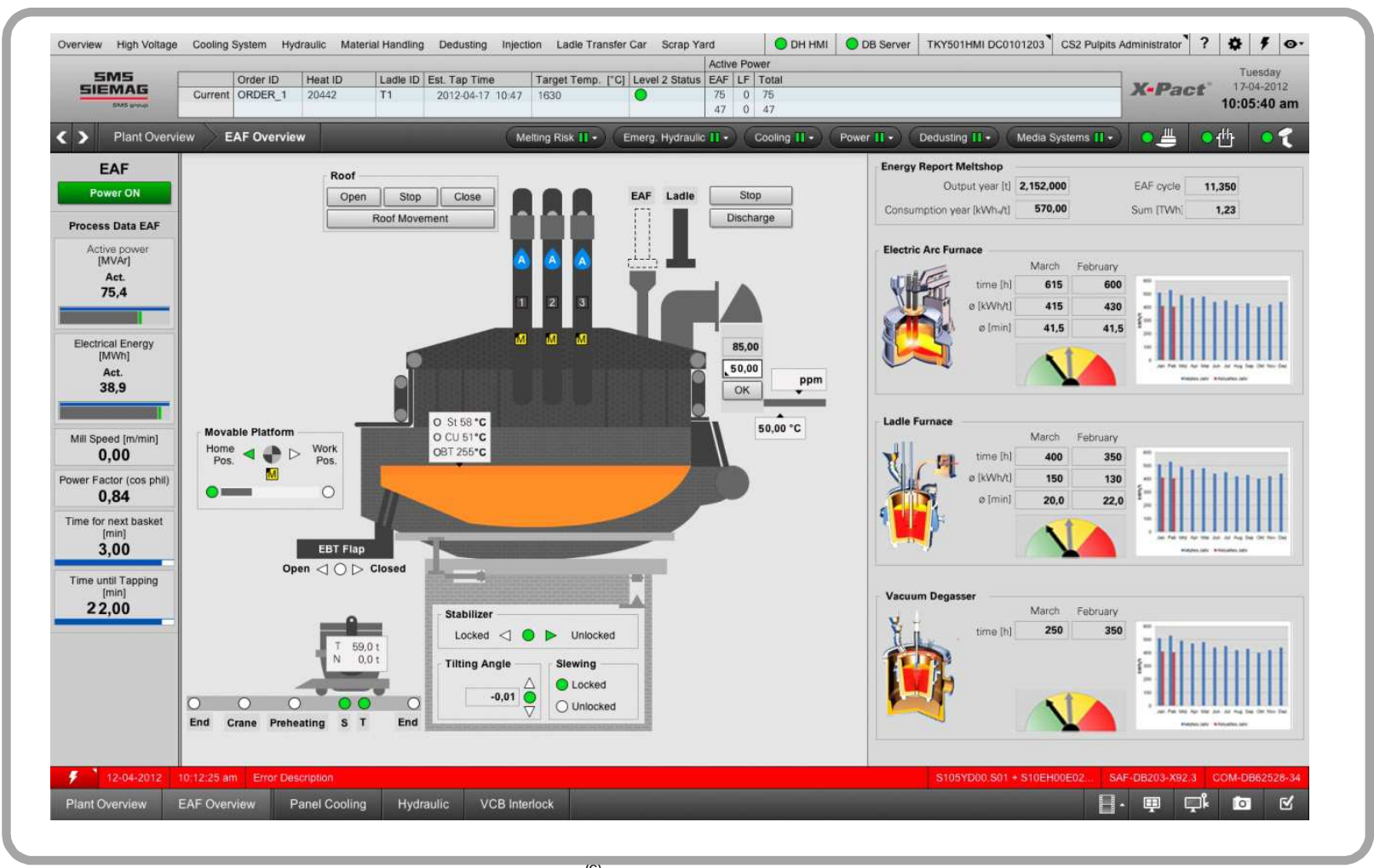

Figure 4: Visualization of the X-Pact ${ }^{(}$Energy Advisor using the example of an EAF

A further component of the Integrated Monitoring System is the module for plant condition monitoring.

\subsection{Plant Condition Module}

The X-Pact ${ }^{\odot}$ Plant Condition Advisor displays the plant status in a transparent manner and makes it possible to optimize the production method. Defined signals and sensor data are monitored and evaluated by intelligent algorithms. From this diagnosis, characteristic values are calculated and displayed as a trend over the time axis. The characteristic values can be monitored with the aid of configurable tolerance bands, within which the characteristic values can fluctuate. Specific alarm messages can be triggered if the values are exceeded.

The display of the evaluation results and the trend displays take place in the system's own HMl but can also, if required, be forwarded to already existing, higher-level maintenance systems. Monitoring allows early recognition of unfavorable operating states or the reaching of wear limits of individual plant components.

On the basis of these results, the plant owner can formulate and implement measures and thus avoid, for instance, unscheduled stoppages with increased repair costs.

The X-Pact ${ }^{\odot}$ Plant Condition Advisor makes available specific functions for the respective plants used in metallurgical engineering, continuous casting, rolling mill engineering and strip processing. In addition, functions for the monitoring of individual components have been implemented, such as for pumps, motors and valves, which are suitable for all plant types. 


\subsubsection{Plant-specific functions}

Functions for continuous casters and for rolling mills are to be presented here as examples.

\subsubsection{Continuous casters}

A selection of the functions of continuous casters is shown in Figure 5 .

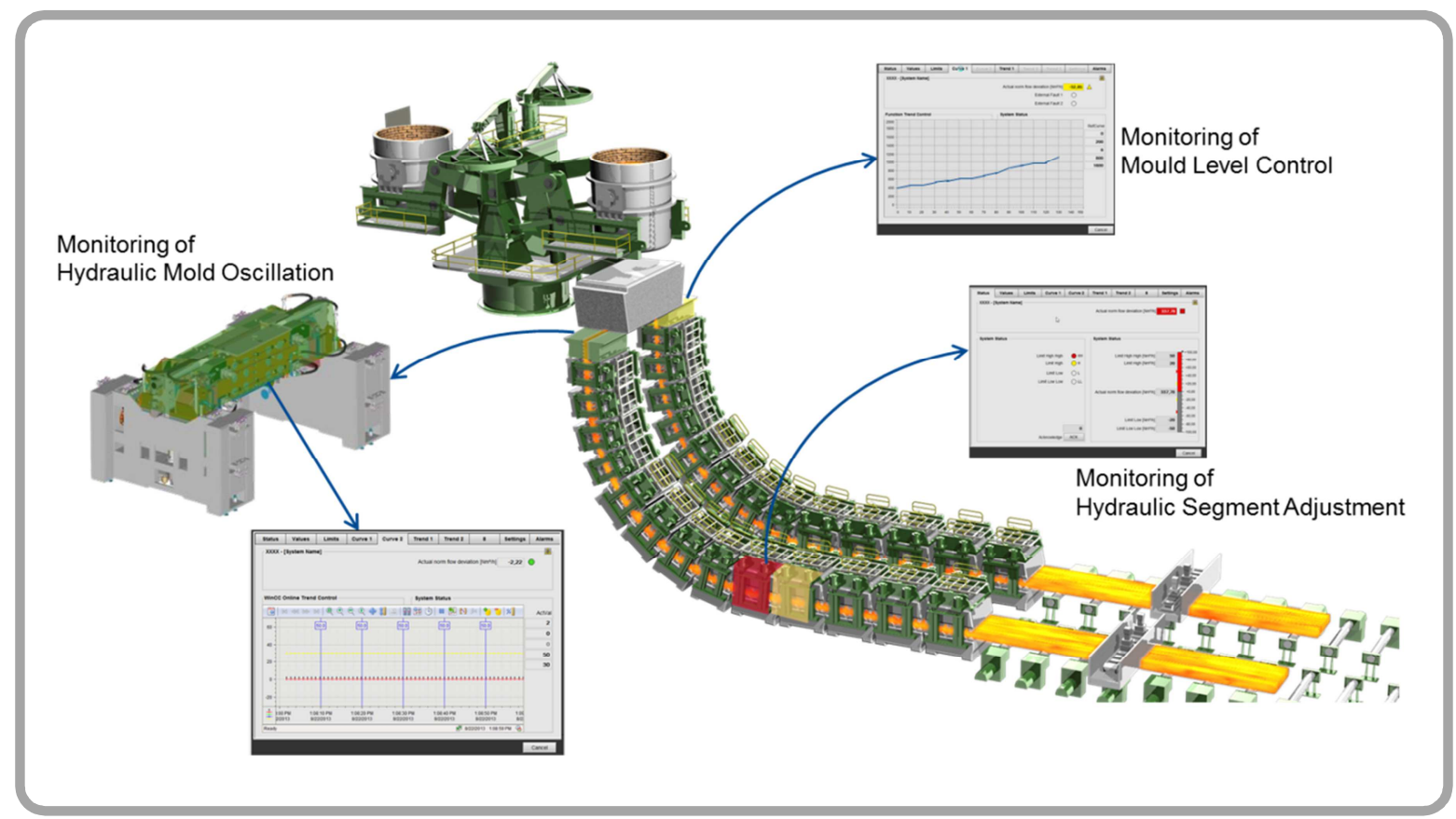

Figure 5: Examples of functions for continuous casters

- Mold level control system - Monitoring of the stopper rod position for its control quality:

Good control of the mold level is very important for strand quality. For example, if the mold level control deteriorates, an increasing number of casting powder inclusions may occur. To prevent this from happening, the control quality of the stopper rod position is determined regularly prior to start of casting with the aid of a step response and the trend of the control response time is monitored.

A step response is a test in which a sudden setpoint change is specified for the system and the actual-value progression of the system is evaluated.

- Hydraulic mold oscillator - Monitoring of the hydraulic cylinders and valves: Progressive wear of the valves or cylinders can be detected via the valve control of the controller already before such wear starts to affect the synchronized cylinder running or cylinder stroke. To do this, the valve control is monitored as a function of the oscillation stroke and oscillation frequency and their mutual synchronized running. If a defined limit value is exceeded, an alarm is triggered.

- Hydraulic segment adjustment:

The purposes of monitoring the hydraulic segment adjustment are the early recognition of impending segment failures, better ability to schedule the segment maintenance and thus the avoidance of production downtimes and quality problems. To do this, the measured values of the pressure sensors are 
checked for plausibility and spikes and jumps in the measured values of the position sensors are evaluated.

\subsubsection{Rolling mills}

Examples of plant-specific functions for rolling mills are shown in Figure 1 and described below.

These functions support the plant owner in his objective of rolling demanding products while observing product quality and process stability.

- Millstand monitoring:

In order to determine the millstand behavior, a test can be performed on the stand condition automatically during calibration of the roll gap. During this test, monitoring takes place of the friction, any distortions occurring in the stand and the deviations in sensitivity of the roll-force sensors. This makes it possible to check whether the maintenance condition of the millstand is adequate for stable rolling of given final dimensions with the specified product quality.

- Testing of step response of servo-controlled mechatronic systems: To determine the wear on servo-controlled mechatronic systems, the dynamic behavior can be tested by means of a step response. Critical changes can be detected by comparing the characteristic values of the step response (control response time, overshoots, etc.) with empirical values. Above all for loopers and hydraulic roll-gap adjustment systems, these tests provide important information on the plant condition.

- Test on looper:

Heightened wear on the looper can impair the process stability and product quality of steel hot strip mills. By regularly passing through a hysteresis loop, a gradual increasing of friction can be recognized. In addition, further mechanical problems which could result in unscheduled stoppages are detected in good time.

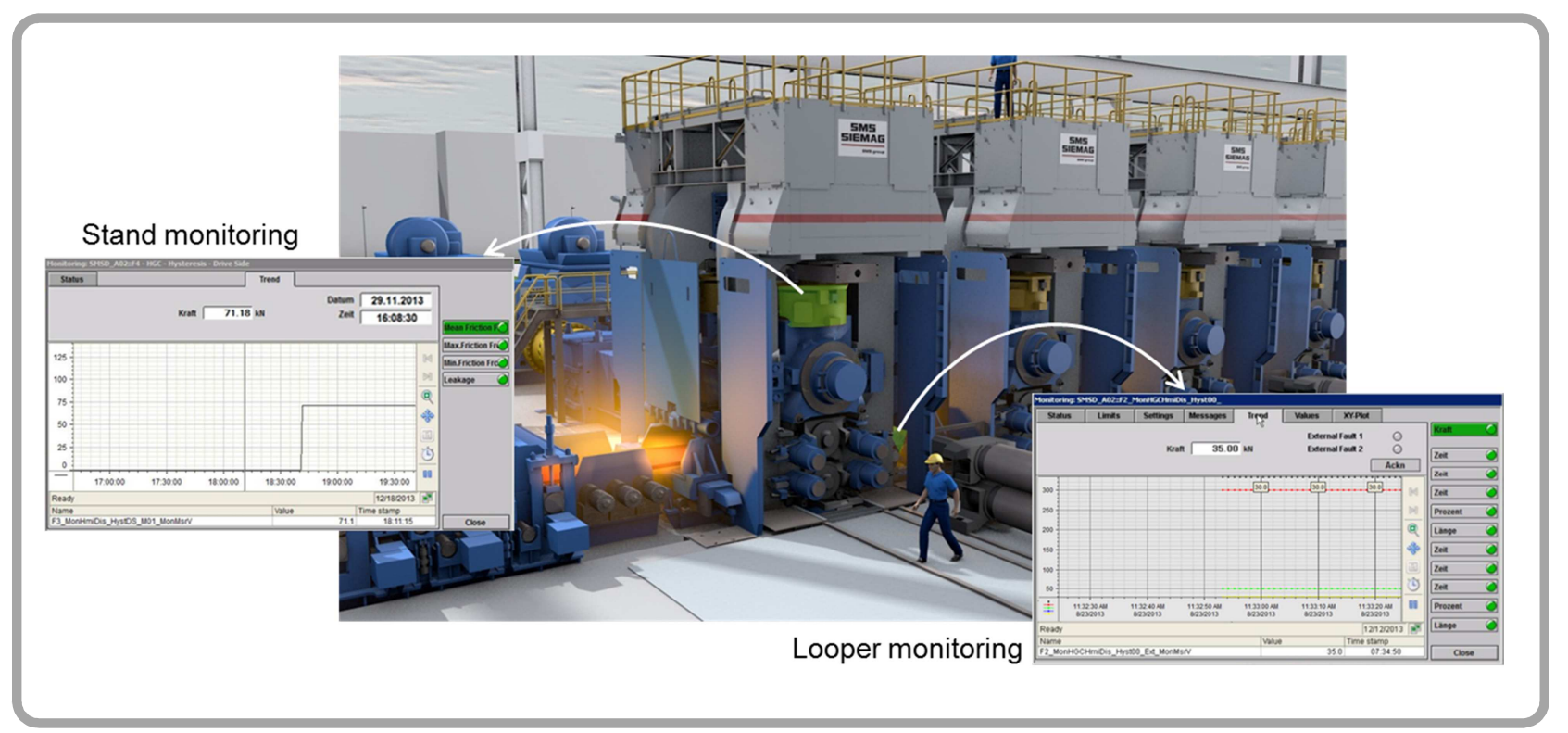

Figure 1: Examples of functions for rolling mills

The examples listed here are only a selection of the implemented functions. Similar functions also exist for other plants, e.g. for metallurgical plants there is monitoring of 
the electrode arm positioning at the EAF or, for strip processing lines, monitoring of the looper cable.

\subsubsection{Component monitoring}

By means of intelligent evaluation algorithms, monitoring can be performed both of electrical components, e.g. intelligent field devices, $1 / O$ modules, controllers and network components, and of mechanical components such as pumps and valves. The following functions, for example, are implemented for component monitoring:

- Motor monitoring

The early detection of faults affecting motors is made possible by monitoring of the power consumption and temperature of motors in combination with the respective production conditions.

- Valve monitoring

Wear on valves can be determined by monitoring the flow rate in relation to the valve opening.

- Flow monitoring

From the ratio of pressure to flow rate it is possible, for example, to monitor the clogging of bottom stirring nozzles at the converter.

The three modules described of the Integrated Monitoring System support the plant owner in the operation and optimization of the integrated plant and allow the production process and plant condition to be examined from various perspectives.

\section{RESULTS AND DISCUSSION}

The modules described support the plant owner in the operation and optimization of the integrated plant and allow the production process and plant condition to be examined from various perspectives.

\section{CONCLUSION}

Owing to the growing pressure of competition, optimized plant operation is becoming an increasingly greater area of priority for plant owners. The Integrated Monitoring System allows a complete view of the overall plant operation. The modules presented here for the monitoring of

- Product quality;

- Energy efficiency;

- Plant condition.

provide the plant owner with tools for improving the product quality and thus for operating the plant with high availability and in the most energy-efficient manner possible.

The Quality Monitoring module allows monitoring of product quality in a manner covering all process stages, furnishes a comparison with the customer's requirements and, with the aid of a quality database, allows long-term storage and analysis of the data.

With the aid of the X-Pact ${ }^{\oplus}$ Energy Advisor, plant operation can be optimized from the point of view of energy-efficient plant operation.

The Plant Condition Advisor contains specific functions for metallurgical plants, continuous casters, rolling mills and strip processing lines, as well as prefabricated intelligent modules for component monitoring. This helps with the performance of preventive maintenance. Furthermore, maintenance costs are saved and the 


\section{9th Industrial $\Pi$ and Automation}

intelligent stocking of spare parts is optimized. In addition, the Plant Condition Advisor supports troubleshooting and thus makes possible an improved plant availability.

The combination of the three modules allows optimized operation of the integrated plant and therefore contributes decisively to cost reduction and quality enhancement.

\section{BIBLIOGRAPHY}

1 Mellenthin, Jesper, Energy Advisor software provides transparency for plants and processes, MPT International, 5/2013,pp. 42-45.

2 Reip, Carl-Peter; Henning, Wolfgang; Kempken, Jens; Krämer, Stephan, Challenges and solutions of compact strip production, MPT International - Metallurgical Plant and Technology, 29, 3/2006, pp.66-68

3 Bruns, M.; Pander, M.; Integrated use of surface quality data in upstream process stages; Conference paper; English; DB-No. 20030207247

4 Iron \& Steel Technology Conference (AISTech), 2014, Indianapolis, USA 